\title{
Role of adenovirus-mediated retinoblastoma 94 in the treatment of human non-small cell lung cancer
}

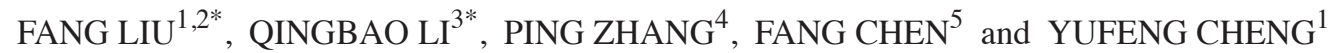 \\ ${ }^{1}$ Department of Radiation Oncology, Qilu Hospital of Shandong University, Jinan, Shandong 250012; \\ ${ }^{2}$ Department of Image, Shandong Medical College, Jinan, Shandong 250002; ${ }^{3}$ Department of Cardiac Surgery, \\ Shandong Provincial Hospital Affiliated to Shandong University, Jinan, Shandong 250021; ${ }^{4}$ Department of Oncology, \\ The People's Hospital of Binzhou City, Binzhou, Shandong 256610; ${ }^{5}$ Department of Ultrasonography, \\ The Second Hospital Affiliated to Shandong University of Traditional Chinese Medicine, \\ Jinan, Shandong 250001, P.R. China
}

Received March 3, 2014; Accepted November 19, 2014

DOI: $10.3892 / \mathrm{mmr} .2015 .3227$

\begin{abstract}
Non-small cell lung cancer (NSCLC) remains the leading cause of cancer-related mortality despite the fact that great advances have been made in therapeutic treatment methods. Therefore, in the present study, the role of adenovirus-mediated retinoblastoma 94 (Ad-RB94) gene therapy in NSCLC was investigated. Following treatment with Ad-RB94, the proportion of A549 cells in the G2/M phase was increased. In the mouse xenograft model, the overexpression of RB94 inhibited the tumor growth compared with the control group and the Ad-LacZ-treated group. In the transplanted tumors, the overexpression of RB94 induced the apoptosis of tumors as well as an increase in the mRNA levels of cyclinB1. In conclusion, the results of the present study suggested that RB94 may effectively inhibit NSCLC tumor cell growth by inducing G2/M cell cycle arrest and apoptosis, indicating that RB94 may be a promising candidate for adjuvant therapy with radiation or chemotherapy in NSCLC.
\end{abstract}

\section{Introduction}

Non-small cell lung cancer (NSCLC) remains the leading cause of cancer-related mortality in China despite the fact that great advances have been made in the therapeutic treatment methods, including surgical resection, chemotherapy and radiotherapy (1). NSCLC mainly comprises two types: adenocarcinoma and squamous cell carcinoma. Two-thirds of

Correspondence to: Dr Yufeng Cheng, Department of Radiation Oncology, Qilu Hospital of Shandong University, 107 West Road, Jinan, Shandong 250012, P.R. China

E-mail: yufengcheng13@126.com

*Contributed equally

Key words: non-small cell lung cancer, retinoblastoma gene, gene therapy, cell cycle arrest
NSCLC patients are diagnosed at a late stage of the disease when the tumors are not resectable. In addition, up to $60 \%$ of all resected patients relapse and succumb to the disease (2). Although radiotherapy has been widely used to treat unresectable and metastatic tumors, radio resistance usually leads to a low overall 5-year survival rate of less than $15 \%$ (3). Therefore, the development of new treatment options including gene therapy is an urgent requirement.

Gene therapy is one of the innovative approaches which have offered new hope for enhancing antitumor effects. The retinoblastoma (RB94) tumor suppressor gene, which lacks the NH2-terminal 112 amino acid residues of the full-length $\mathrm{RB}$ protein (RB110), has been identified to have marked tumor suppressor efficacy compared with wild-type RB110 $(4,5)$. The RB94 protein has been reported to remain in a hypo-phosphorylated state in transfected tumor cells and demonstrate a significantly longer half-life than the RB110 protein $(4,5)$. In addition, RB94 exhibits an increased antitumor efficacy in RB-negative and RB-positive human tumors, including fibrosarcoma and bladder, lung and prostate carcinoma, while the effects of RB110 are limited to RB-negative tumors (4). Preclinical studies also demonstrate that adenovirus-mediated (Ad)-RB94 gene transfer significantly suppresses the growth of human head and neck cancer, bladder carcinoma, pancreatic carcinoma and esophageal cancer in vitro and in vivo (6-9). Moreover, the combination of RB94 and radiation therapy (XRT) results in synergistic tumor growth suppression of head and neck squamous cell carcinoma (10). Therefore, RB94 appears to be a promising candidate for gene therapy in NSCLC.

In the present study, an adenoviral vector carrying the RB94 gene (Ad-RB94) was constructed. Subsequently, we investigated whether Ad-RB94 exerted therapeutic effects in NSCLC as well as examining the underlying molecular mechanisms.

\section{Materials and methods}

Cell culture. The human NSCLC cell line A549 has been well characterized and is known to express wild-type RB (11). The 
A549 cells were cultured in RPMI 1640 (Hyclone, Logan, UT, USA) with $10 \%$ fetal bovine serum at $37^{\circ} \mathrm{C}$ in $5 \% \mathrm{CO}_{2}$.

Construction of recombinant adenoviral vectors. The construction of recombinant adenovirus vector for the RB94 gene was carried out using Gateway ${ }^{\mathrm{TM}}$ clone technology (GeneSil, Wuhan, China). Briefly, total RNA was extracted from a human embryo and reversed transcribed to obtain object cDNA, then the hRB94 gene fragment was amplified by polymerase chain reaction (PCR). The attB-flanked PCR primers were designed and used to amplify the hRB94 gene by PCR. An entry clone was performed by a BP recombination reaction with attB-PCR products and donor vector pDONRTM221. Then the entry clone and the target vector $\mathrm{Ad} / \mathrm{CMV} / \mathrm{V} 5-\mathrm{DEST}$ with attR1 and attR2 sites was recombined in vivo to create the expression clone (Ad-hRB94) using an efficient LR recombination reaction. Next, the expression clone was confirmed by PCR and sequencing. Ad-hRB94 was digested with Pac I and transferred into 293A cells to be packaged into adenovirus stock. Ad-hRB94 was amplified by infection of $293 \mathrm{~A}$ cells and the titer was measured. Ad-LacZ, a replication-defective control adenovirus not carrying the RB94 gene, was obtained from Invitrogen Life Technologies (Carlsbad, CA, USA). The viruses were amplified and plaque-purified. Titers were determined by standard plaque assays.

Western blot analysis. The cells were lysed by adding $100 \mu 1$ radioimmunoprecipitation assay lysis buffer [1\% Triton X-100, $1 \%$ deoxycholate, $0.1 \%$ sodium dodecyl sulfate (SDS), $1 \mathrm{mM}$ phenylmethylsulfonyl fluoride] purchased from Beyotime Institute of Biotechnology (Shanghai, China) and then quantitated using a bicinchoninic acid (BCA) assay kit with phosphate-buffered saline (PBS) as a standard (Pierce, Rockford, IL, USA). Equal quantities of protein $(50 \mu \mathrm{g})$ from the different cells were separated by $10 \%$ SDS-polyacrylamide gel electrophoresis. The separated proteins were transferred onto nitrocellulose (GE Healthcare, Buckinghamshire, UK). Non-specific binding sites were blocked using PBS-Tween-20 and $5 \%$ non-fat dried milk for $1 \mathrm{~h}$ at room temperature. Following blocking, the samples were incubated overnight at $4^{\circ} \mathrm{C}$ with primary antibody mouse-antihuman $\mathrm{RB}$ (BD Biosciences Pharmingen, San Diego, CA, USA), which recognizes both full-length RB and NH2-terminal-truncated RB94 proteins, at a concentration of 1:200 in Tris-buffered saline-Tween-20. Membranes were washed three times for 10 min in a buffer containing PBS and $0.1 \%$ Tween-20, and then incubated with an appropriate goat antimouse secondary antibody conjugated to horseradish peroxidase (Amersham Pharmacia Biotech, Piscataway, NJ, USA) applied for $1 \mathrm{~h}$ at room temperature. Reactive bands were detected with the ECL chemiluminescence reagent (Amersham Pharmacia Biotech, Freiburg, Germany). $\beta$-actin was detected using monoclonal anti- $\beta$-actin (Sigma, St. Louis, MO, USA).

Reverse transcription-quantitative PCR (RT-qPCR). Total mRNA was extracted from tumors in each of the studied groups with an mRNA isolation kit (Takara, Dalian, China), and $3 \mu \mathrm{g}$ total RNA was used for cDNA synthesis by SuperScript II reverse transcriptase (Invitrogen) according to the standard instructions. qPCR was performed in a final volume of $20 \mu \mathrm{l}$ containing $1 \mu \mathrm{l}$ each cDNA template, $2 \mu \mathrm{l}$ each $10 \mathrm{nM}$ primer (RB94 F: 5'-GAA TCT GCT TGT CCT CTT AAT CTT AAT CTT CC-3' and R: 5'-GAA GAT GGT GAT GGG ATT TC-3'; cyclinB1 F: 5'-CAG TCA GAC CAA AAT ACC TAC TGG GT-3' and R: 5'-ACA CAA ACC AGC TGC AGC ATC TTC TT-3'), and $10 \mu 1$ SYBR-Green Master mix. Quantification was carried out using the comparative cycle threshold $(\mathrm{Ct})$ method, and water was used as the negative control. Ct values were calculated by determining the cycle number at which the fluorescence exceeded the threshold limit. The average $\mathrm{Ct}$ values for the target gene were normalized to an endogenous housekeeping gene encoding 18S rRNA.

Flow cytometric analysis. Cells were seeded at a density of $1 \times 10^{6}$ cells/well in six-well tissue culture plates and allowed to adhere overnight. Media were removed and cells were incubated with either Ad-RB94 or Ad-LacZ at a multiplicity of infection of 10, or control with PBS in $5 \mathrm{ml}$ media for $2 \mathrm{~h}$, after which $10 \mathrm{ml}$ media was added. After $24 \mathrm{~h}$, harvested cells were trypsinized, washed with PBS, fixed in suspension in $75 \%$ ethanol, and kept at $-20^{\circ} \mathrm{C}$ until analysis. Fixed cells were centrifuged and resuspended in PBS. After adding RNase $(1 \mathrm{mg} / \mathrm{ml})$ to the cell suspension, the cells were incubated for $30 \mathrm{~min}$ at $37^{\circ} \mathrm{C}$, and finally stained with propidium iodide $(50 \mu \mathrm{g} / \mathrm{ml})$ for $1 \mathrm{~h}$ at $4^{\circ} \mathrm{C}$ and then analyzed with FACScan. Cell distribution was analyzed by WinMDI 2.8 software (Joseph Trotter, San Diego, CA, USA).

Xenograft tumor in vivo. Eighteen six-week-old female $\mathrm{BALB} / \mathrm{c}$ nude mice were injected subcutaneously into the posterior flank region with $5 \times 10^{6} \mathrm{~A} 549$ cells in $100 \mu 1$ normal saline. After 10 days, skin flaps were raised and tumors were exposed. Then mice were divided into three groups randomly: a control group, an Ad-LacZ group and an Ad-RB94 group ( $\mathrm{n}=6$ for each). The tumors were injected intratumorally on day $0,4,7$ and 14 following the establishment of the groups. The mice were sacrificed and the tumors were measured using calipers on day 21 . The volumes were calculated according to the formula: $\mathrm{V}\left(\mathrm{mm}^{3}\right)=$ larger diameter $(\mathrm{mm}) \mathrm{x}$ smaller diameter ${ }^{2}\left(\mathrm{~mm}^{2}\right) \times \pi / 6$. Institutional guidelines with regard to animal experimentation and care were followed for the nude mice during the experiment.

Apoptosis detection by Hoechst staining. The apoptosis of tumors was evaluated by Hoechst staining according to the manufacturer's instructions. First, paraffin sections were deparaffinized, washed once with PBS for $10 \mathrm{~min}$ and stained with Hoechst 33258 (Beyotime Institute of Biotechnology) for $5 \mathrm{~min}$. Then they were given neutral gummi and observed by fluorescent Olympus microscopy. Four hundred cells were counted in four different visual fields for every slide. The percentage of apoptotic positive cells was calculated by counting the positive cells and the total number of cells from every visual field.

Statistical analysis. All the results are expressed as the means \pm standard deviation. Statistical significance was determined using SPSS 17.0 for Windows (SPSS, Inc., Chicago, 
A

\section{RB94}

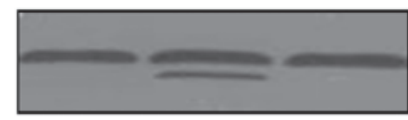

$110 \mathrm{KDa}$

$94 \mathrm{KDa}$

Actin

B

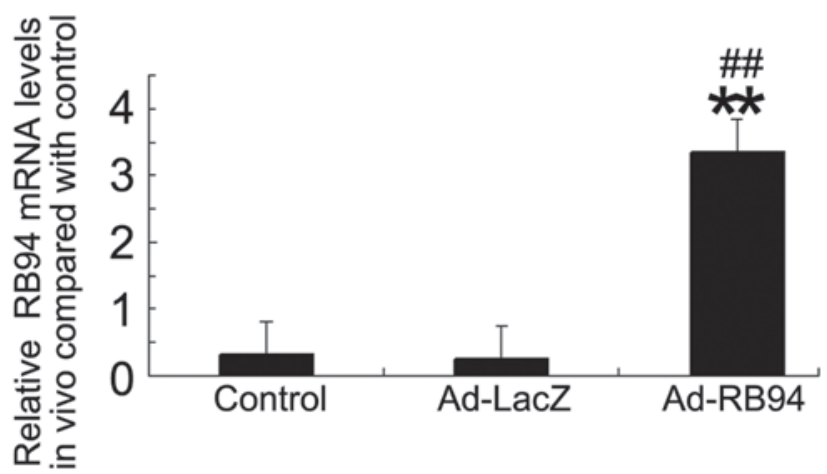

Figure 1. Expression of retinoblastoma (RB) in vitro and in vivo. (A) Detection of RB94 in A549 cells by western blot analysis. (B) Reverse transcriptionquantitative polymerase chain reaction analysis of RB94 mRNA levels in vivo. Data are expressed as the means $\pm \mathrm{SD}(\mathrm{n}=3)$. ${ }^{* * *} \mathrm{P}<0.01$ compared with control; ${ }^{\# \#} \mathrm{P}<0.01$ compared with Ad-LacZ group.

IL, USA). One-way analysis of variance was performed for multiple comparisons followed by Fisher least significant difference post hoc comparisons. $\mathrm{P}<0.05$ was considered to indicate a statistically significant difference.

\section{Results}

Expression of RB94 following Ad-RB94 transfection. Following Ad-RB94 transfection, western blot analysis revealed two bands at $110 \mathrm{kDa}$ (wild-type $\mathrm{RB}$ ) and $94 \mathrm{kDa}$ (RB94) in the A549 cells (Fig. 1A) with the RB antibody. However, the control and Ad-LacZ-transfected A549 cells only expressed wild-type RB protein at $110 \mathrm{kDa}$. Next, the expression of RB94 in vivo was determined by RT-qPCR. As shown in Fig. 1B, the in vivo RB94 mRNA level was higher in the Ad-RB94 group than in the Ad-LacZ and control groups. There was no significant difference between the control and the Ad-LacZ group.

Ad-RB94 induces G2/M cell cycle arrest. As shown in Fig. 2, flow cytometry revealed that overexpression of Ad-RB94 increased the proportion of A549 cells in the G2/M phase, whereas it decreased the proportion of A549 cells in the G0/G1 phase and the $\mathrm{S}$ phase, indicating that Ad-RB94 may induce G2/M cell cycle arrest in A549 cells.

RB94 transfer inhibits tumors growth and cyclinB1 $\mathrm{mRNA}$ levels in vivo. Following the 21 days of treatment, mice were sacrificed and the volumes of tumors were examined. Treatment with Ad-RB94 significantly inhibited the growth of tumors compared with the control and Ad-LacZ. There was no significant difference between the control and Ad-LacZ groups (P>0.05; Fig. 3A). The cyclinB1 mRNA level of the

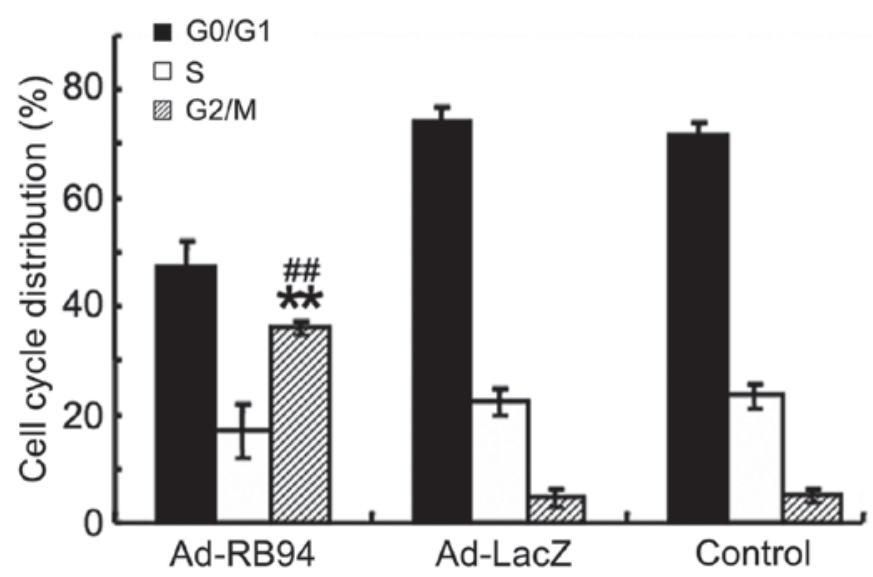

Figure 2. Retinoblastima (RB) 94 gene transfer induces G2/M cell cycle arrest in vitro. The experiment was replicated three times. Data are expressed as the means $\pm \mathrm{SD}(\mathrm{n}=3)$. ${ }^{* *} \mathrm{P}<0.01$ compared with control; ${ }^{\# \#} \mathrm{P}<0.01$ compared with Ad-LacZ group.

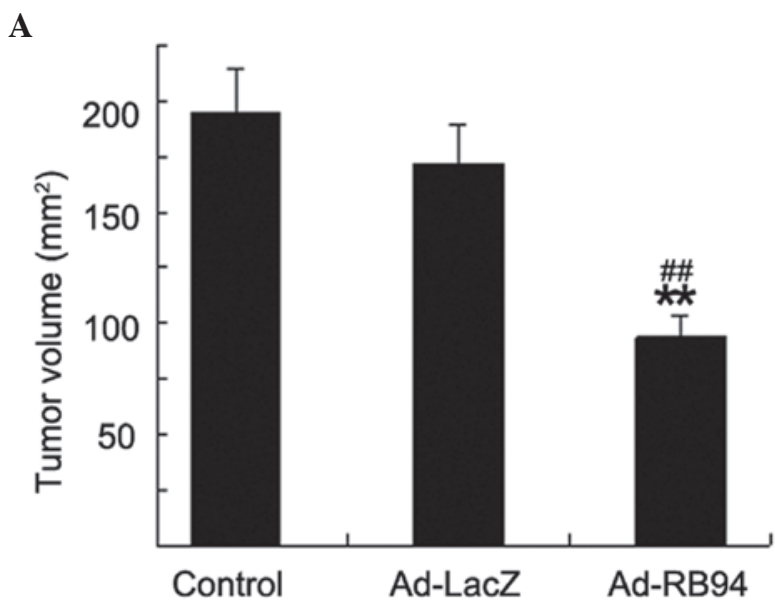

B

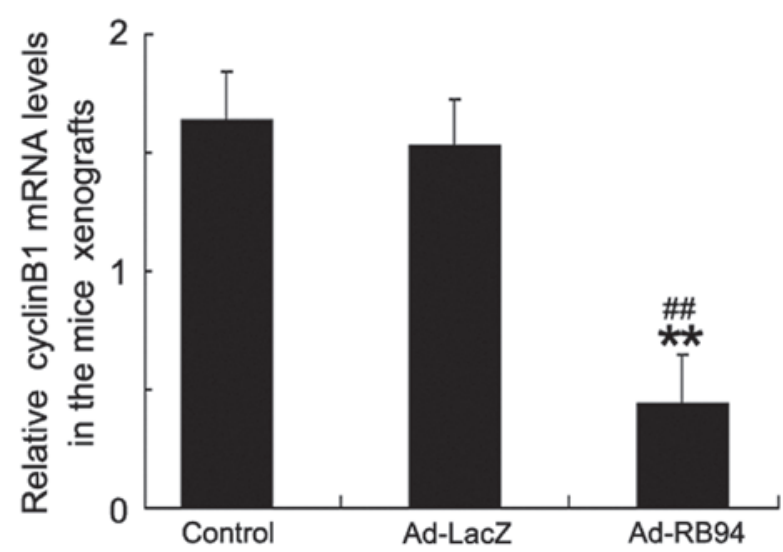

Figure 3. Retinoblastima (RB) 94 transfection suppresses tumor growth in vivo. (A) Tumor volumes following treatment. (B) Relative cyclinB1 mRNA in the tumor following RB94 treatment. Data are expressed as the means $\pm \mathrm{SD}(\mathrm{n}=6) .{ }^{* *} \mathrm{P}<0.01$ compared with control; ${ }^{\# \#} \mathrm{P}<0.01$ compared with Ad-LacZ group.

tumors following Ad-RB94 treatment decreased significantly compared with those of the Ad-LacZ and the control groups (Fig. 3B). There were no significant differences in the levels 


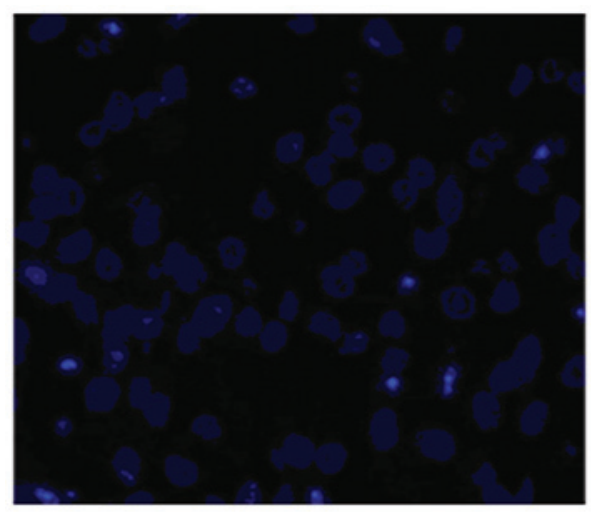

Control

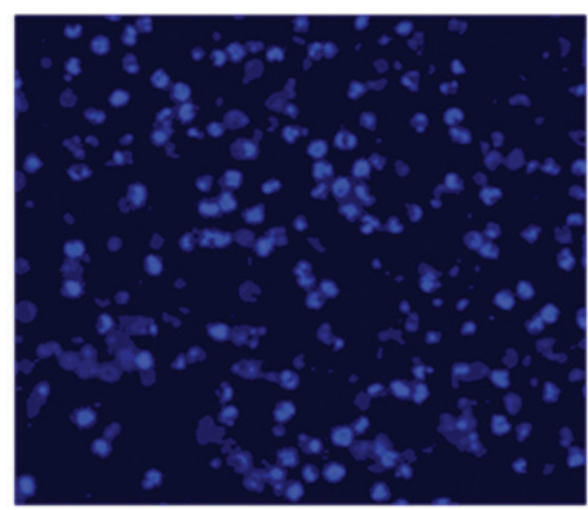

Ad-RB94

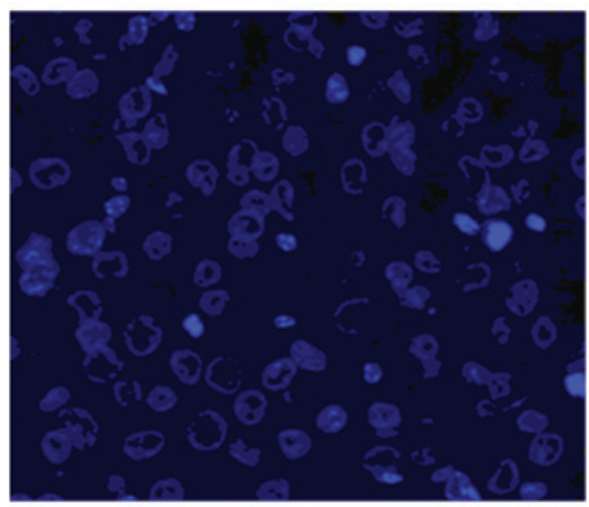

Ad-lacZ

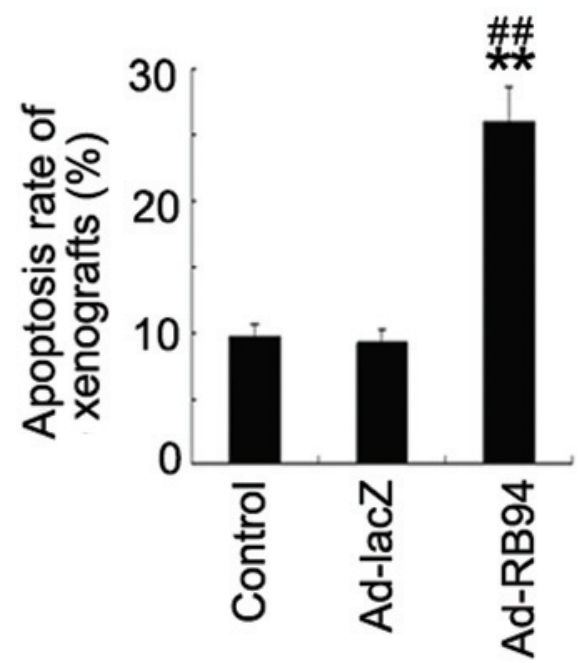

Figure 4. Retinoblastima (RB) 94 transfection induces tumor apoptosis in vivo. Data are expressed as the means $\pm \mathrm{SD}(\mathrm{n}=6)$. ${ }^{* *} \mathrm{P}<0.01$ compared with control; ${ }^{\# \#} \mathrm{P}<0.01$ compared with Ad-LacZ group.

of cyclinB1 mRNA between the Ad-LacZ and the control groups.

Apoptosis detection in vivo. To further investigate whether Ad-RB94 induced NSCLC apoptosis in vivo, an apoptosis detection kit was used to determine apoptosis-related molecular markers in tumor sections (Fig. 4). The results demonstrated that treatment with Ad-RB94 induced an apoptosis rate of $26 \%$. However, only a small number of positive cells were observed in the control and the Ad-LacZ-treated mice.

\section{Discussion}

The results of the present study demonstrate that RB94 overexpression induces the G2/M cell cycle arrest of NSCLC in vitro. Overexpression of RB94 in vivo also induced the apoptosis of NSCLC cells and decreased the tumor cyclinB1 levels, which may cause G2/M phase block $(12,13)$, and thereby inhibited the growth of the transplanted tumor.

It has been reported that RB94 is capable of inducing apoptosis in human pancreatic tumors and in head and neck cancer $(6,8,10)$. Ad-RB94 transfection also induces cell cycle arrest in the $\mathrm{G} 2 / \mathrm{M}$ phase and decreases telomerase activity in human head and neck squamous cell carcinoma (6). In the present study, the results demonstrated that Ad-RB94 transfection induced the G2/M phase block in the A549 lung cancer cells. The in vivo investigation also suggested that Ad-RB94 transfection decreased the levels of cyclinB1. The Cdk1/cyclinB1 complex plays a significant role in the progression of the cell cycle from the $\mathrm{G} 2$ to $\mathrm{M}$ phase, and downregulating cyclinB1 could cause $\mathrm{G} 2 / \mathrm{M}$ phase block $(12,13)$. Thus, these data indicated that the overexpression of Ad-RB94 may result in G2/M phase block in lung cancer cells. In the tumor xenograft study, the present study confirms that an adenoviral vector efficiently delivers the RB94 gene into targeted cells and suppresses the tumor cell growth. RB94 expression in the NSCLC tumor cells leads to apoptotic tumor cell death in the xenograft nude mouse model.

In conclusion, the present study provided evidence that RB94 significantly inhibits the growth of NSCLC cells in vitro and in vivo. These effects are elicited by arresting the cells at the G2/M phase and triggering apoptosis of the tumor cells. These results suggest that RB94 is a promising candidate for adjuvant therapy with radiation or chemotherapy, as tumor cells are most sensitive to radiation or cytotoxic drugs in 
this cell cycle phase. Further research is required to better elucidate the molecular pathways underlying these effects of RB94.

\section{References}

1. Goya T, Asamura H, Yoshimura H, et al: Prognosis of 6644 resected non-small cell lung cancers in Japan: a Japanese lung cancer registry study. Lung Cancer 50: 227-234, 2005.

2. Pisters KM, Evans WK, Azzoli CG, et al: Cancer Care Ontario and American Society of Clinical Oncology adjuvant chemotherapy and adjuvant radiation therapy for stages I-IIIA resectable non small-cell lung cancer guideline. J Clin Oncol 25: 5506-5518, 2007.

3. Erridge SC, Moller H, Price A and Brewster D: International comparisons of survival from lung cancer: pitfalls and warnings. Nat Clin Pract Oncol 4: 570-577, 2007.

4. Xu HJ, Zhou Y, Seigne J, et al: Enhanced tumor suppressor gene therapy via replication-deficient adenovirus vectors expressing an N-terminal truncated retinoblastoma protein. Cancer Res 56 2245-2249, 1996.

5. Xu HJ, Xu K, Zhou Y, Li J, Benedict WF and Hu SX: Enhanced tumor cell growth suppression by an $\mathrm{N}$-terminal truncated retinoblastoma protein. Proc Natl Acad Sci USA 91: 9837-9841, 1994.

6. Li D, Day KV, Yu S, et al: The role of adenovirus-mediated retinoblastoma 94 in the treatment of head and neck cancer. Cancer Res 62: 4637-4644, 2002.
7. Zhang X, Multani AS, Zhou JH, et al: Adenoviral-mediated retinoblastoma 94 produces rapid telomere erosion, chromosomal crisis, and caspase-dependent apoptosis in bladder cancer and immortalized human urothelial cells but not in normal urothelial cells. Cancer Res 63: 760-765, 2003.

8. Roig JM, Molina MA, Cascante A, et al: Adenovirus-mediated retinoblastoma 94 gene transfer induces human pancreatic tumor regression in a mouse xenograft model. Clin Cancer Res 10: 1454-1462, 2004.

9. Zhang H, Li J, Wang YY, et al: Retinoblastoma 94 enhances radiation treatment of esophageal squamous cell carcinoma in vitro and in vivo. J Radiat Res 53: 117-124, 2012.

10. Araki K, Ahmad SM, Li G, et al: Retinoblastoma RB94 enhances radiation treatment of head and neck squamous cell carcinoma. Clin Cancer Res 14: 3514-3519, 2008

11. Edelman MJ, Quam H and Mullins B: Interactions of gemcitabine, carboplatin and paclitaxel in molecularly defined non-small-cell lung cancer cell lines. Cancer Chemother Pharmacol 48: 141-144, 2001.

12. Vairapandi M, Balliet AG, Hoffman B and Liebermann DA: GADD45b and GADD45g are cdc2/cyclinB1 kinase inhibitors with a role in $\mathrm{S}$ and $\mathrm{G} 2 / \mathrm{M}$ cell cycle checkpoints induced by genotoxic stress. J Cell Physiol 192: 327-338, 2002.

13. Gomathinayagam R, Sowmyalakshmi S, Mardhatillah F, Kumar R, Akbarsha MA and Damodaran C: Anticancer mechanism of plumbagin, a natural compound, on non-small cell lung cancer cells. Anticancer Res 28: 785-792, 2008. 